\title{
Many electron effects in semiconductor quantum dots
}

\author{
R K PANDEY*, MANOJ K HARBOLA, V RANJAN and VIJAY A SINGH \\ Physics Department, Indian Institute of Technology, Kanpur 208 016, India
}

\begin{abstract}
Semiconductor quantum dots (QDs) exhibit shell structures, very similar to atoms. Termed as 'artificial atoms' by some, they are much larger $(1100 \mathrm{~nm})$ than real atoms. One can study a variety of manyelectron effects in them, which are otherwise difficult to observe in a real atom. We have treated these effects within the local density approximation (LDA) and the Harbola-Sahni (HS) scheme. HS is free of the selfinteraction error of the LDA. Our calculations have been performed in a three-dimensional quantum dot. We have carried out a study of the size and shape dependence of the level spacing. Scaling laws for the Hubbard ' $U$ ' are established.
\end{abstract}

Keywords. Quantum dot; Hubbard ' $U$ '; Coulomb blockade.

\section{Introduction}

Powerful single-electron experimental spectroscopic techniques available now can resolve the discrete level structures of quantum dots (Ashoori 1996). The electronelectron interaction plays a very important role in them (Ashoori 1996; Klein et al 1996, 1997; Tarucha et al 1996). Our calculations involve multielectron effects. These effects have been treated earlier mainly within the local density approximation (LDA) of the density functional theory (DFT) (Macucci et al 1995, 1997). The selfinteraction error in the LDA may have significant effect on these results (Macucci et al 1995, 1997). In addition to LDA we have also employed the Harbola-Sahni (HS) scheme which treats the exchange potential essentially exactly and is free of the self-interaction error of the LDA (Harbola and Sahni 1989).

We have considered a 3-D quantum well in our calculations. Effective mass theory (EMT) calculations on QD have traditionally adopted either a square well or a parabolic shape for the confining potential. It is customary to model the QD by a square well potential with finite/ infinite barrier while studying band gap enhancement and excitonic effect (Singh et al 2000). On the other hand, researchers performing Coulomb blockade calculations routinely choose parabolic confinement (Macucci et al 1995, 1997). There is no a priori justification for either. It is, therefore, important to investigate the role of confinement potential and its influence on Coulomb blockade.

Extensive calculations have been conducted for the size and shape dependence of the capacitive energy and a comparison of the LDA and HS is made. Charge density and the effective potential are also calculated. We have found that Janak's theorem provides a more economic

*Author for correspondence route than the conventional one for the calculation of the capacitive energy. The relationship between the Hartree, exchange and correlation energy terms is explored and the effect of barrier height on the capacitive energy has been calculated. These results are presented elsewhere. In this paper we have reported the dependence of the capacitive energy on size of the QD and shape of the confining potential, shape dependence of the level spacing and the electron-electron interaction.

\section{Model}

We have solved the Schrödinger equation self-consistently within the effective mass theory (EMT) approximation. We have included electron-electron interaction effect via a local density functional approach. The Hamiltonian of the system is given by the following expression

$$
H=-\sum_{i=1}^{N} \frac{1}{2 m^{*}} \nabla_{i}^{2}+\frac{1}{2} \sum_{i=1}^{N} \sum_{j \neq 1}^{N} \frac{1}{\varepsilon\left|\vec{r}_{i}-\vec{r}_{j}\right|}+V_{\mathrm{ext}}(r) .
$$

The above Hamiltonian is written in Hartree's atomic unit, where $\hbar=m_{\mathrm{e}}=e=1$ and unit of energy is $27.2 \mathrm{eV}$ and that of length (size) is $0.53 \AA$. In the above Hamiltonian, $m^{*}$ is the effective mass of the electron inside the QD in units of $m_{\mathrm{e}}$, the free electron mass, and $\varepsilon$ the relative dielectric constant of the material. The first term in the above Hamiltonian is the kinetic energy of electrons, the second term is the electron-electron interaction energy and the last term is the external potential for an $N$ electron system. The above many-body Hamiltonian can be reduced to a single particle Schrödinger type Hamiltonian for the ground state within the density functional theory (DFT) of Kohn and Sham (1965). The equation obtained is called the Kohn-Sham equation (Kohn and Sham 1965). 
Our main thrust is on studying the dependence of the capacitance on the shape of the external potential. Almost all reported works have used a harmonic potential. There is no specific reason for this choice except for the computational ease. On the other hand most of the work on band gap enhancement based on EMT have adopted a square well potential. The form of the external potential we have used interpolates smoothly between the two shapes:

$$
V_{\text {ext }}(r)=\frac{V_{0}}{R^{k}} r^{k}-V_{0},
$$

here $V_{0}$ is the depth of the potential. This can be given by the conduction band offset (valence band offset) between the QD and the surrounding layer for the electron (hole). $R$ is the radius of the QD. The external potential will take different shapes as $k$ assumes positive integral values from 1 to $\infty$. In particular, $k=2$ is the harmonic confinement case and $k \rightarrow \infty$ is the square well case. From simple electrostatics, it is evident that the harmonic case, $k=2$ corresponds, to a good approximation, to a uniform positive charge distribution over a sphere. The triangular potential may arise due to a constant electric field. Quartic $(k \geq 4)$ and higher order terms arise when there is an excess positive charge near the surface.

\section{Results}

The capacitive energy is calculated as (Iafrate et al 1995)

$$
\begin{aligned}
\frac{e^{2}}{C(N)} & =E(N+1)-2 E(N)+E(N-1), \\
& =I(N)-A(N),
\end{aligned}
$$

where $C(N)$ and $E(N)$ are the capacitance and the ground state energy of a system with $N$ electrons within DFT, $I(N)$ the ionization potential, $A(N)$ the electron affinity. The ionization potential and electron affinity can in principle be determined through the photoemission and inverse photoemission experiments, respectively. Their difference may be taken to be a measure of the ' $U$ ' term of the Hubbard model. We shall call it, $U_{\text {eff. The Hubbard }}$ ' $U$ ' in extended systems is independent of the electron filling. In our case, $U_{\text {eff }}$ depends both on QD size and number of electrons. Our calculations have been done for neutral system.

In figure 1, we have depicted the capacitive energy, $e^{2} / C(N)$ as a function of the total number of electrons $(N)$ for different $\mathrm{QD}$ sizes $(R), R=4$ and $R=8$, with harmonic confinement $(k=2)$ for both the HS and the LDA. We see that the capacitive energy is larger for smaller QD. This is due to the larger confinement of the charges inside the smaller QD and the consequent large charge density. Thus putting an extra electron requires large energy. We also observe peaks in the capacitive energy. These peaks are due to the shell filling effect. The shell filling effect is observed at $N=2$ and 8 for $R=4$ and $N=2,8,18$ and 20 for $R=8$.

We also notice that there is very little difference in capacitive energy calculated using the HS scheme and the LDA. But the difference is larger for smaller $N$ or when the degeneracy is lifted. This is shown in the inset of figure 1. HS has self-interaction correction built in it and hence calculates very good eigen values and total energies for a single electron system, whereas LDA is known to yield poor results for a single electron system due to the self-interaction of the electron. Nearly exact exchange treatment of HS is responsible for this large difference at smaller $N$. The perturbation potential responsible for removing the degeneracy is $V_{\mathrm{H}}+V_{\mathrm{xc}}$. These are $V_{\mathrm{H}}+V_{\mathrm{xc}}^{\mathrm{LDA}}$ and $V_{\mathrm{H}}+V_{x}^{\mathrm{HS}}$, respectively for LDA and $\mathrm{HS}$ schemes and they are very different from one another. The levels where degeneracy is removed, is like a closed shell structure and the small number of electrons are like valence electrons in the close shell atom. Hence once again the system is like the one with small number of electrons. Thus self-interaction of electrons causes difference to appear between the LDA and the HS scheme. This difference is more pronounced when the number of electrons is small. We also observe that a smaller dot accommodates less number of electrons in comparison to the larger one. We find that HS scheme usually binds more electrons than LDA. We have also shown the capacitive energy calculated using the more economic route, the Janak's theorem,

$$
\frac{e^{2}}{C(N)}=\varepsilon_{\max }(N+1)-\varepsilon_{\max }(N),
$$

where $\varepsilon_{\max }(N)$ is the energy eigen value of the highest occupied orbital of an $N$-electron system.

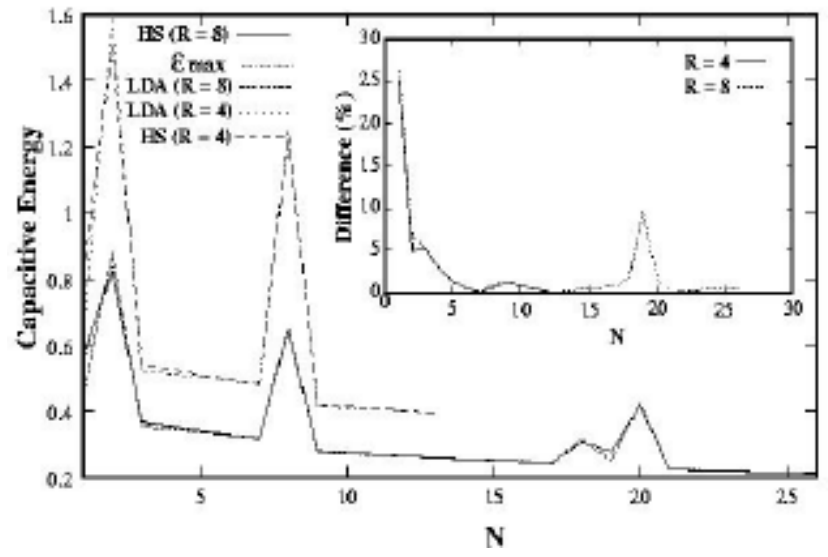

Figure 1. Capacitive energy, $e^{2} / C(N)$, as a function of total number of charges, $N$. 
The work reported henceforth (figures 2-6) is based on the HS (Harbola-Sahni) scheme. In figure 2, we show the capacitive energy $\left(e^{2} / C(N)\right)$ as a function of the total number of electrons $(N)$ for $k=1,2,4$ and size $R=4$. In the inset a similar plot is shown for $k=4,8$ and 10. We have described earlier that changing $k$ means change in the shape of the external confining potential. Thus $k=1$ represents a quasi-triangular confinement, $k=2$ a quasiharmonic confinement and as we approach large value of $k$, e.g. $k=8$ or 10 , the potential can be considered as a quasi-square well confinement.

The capacitive energy is always less for a given number of electrons when $k$ is larger. This is because when $k$ is larger the confinement is reduced and the effective size of the QD is larger. Semiclassically $C(N) \sim R_{\text {eff }}$ and hence the capacitive energy $\sim 1 / R_{\text {eff. }}$ We also observe that the shell structure of these systems change as shape of the potential is changed. As the shape becomes more and more quasi-square, the shell structure remains the same

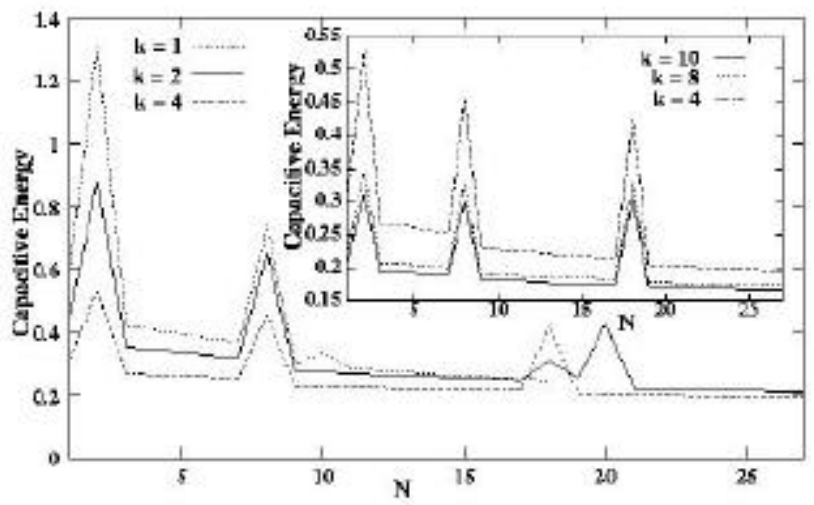

Figure 2. Capacitive energy, $e^{2} / C(N)$ as a function of total number of charges $N$ for $k=2$.
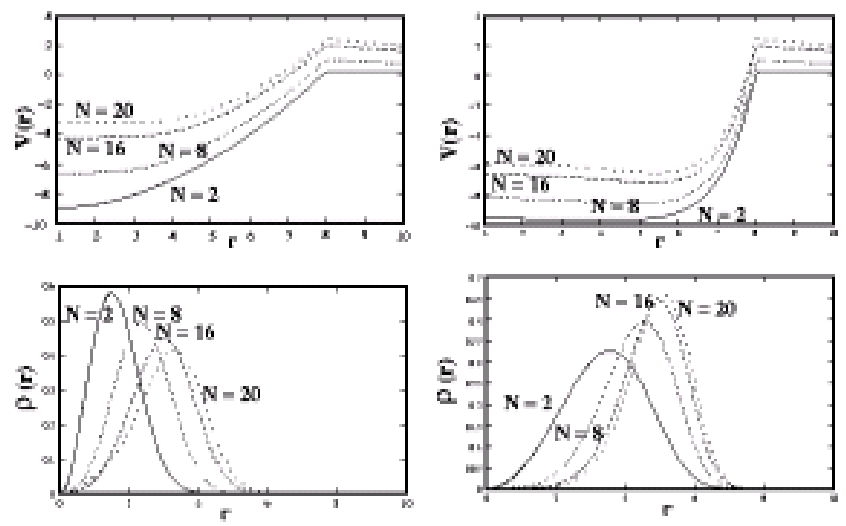

Figure 3. On the top left is depicted the effective potential for $k=2$ with $N=2,8,16$ and 20 electrons. The effective potential for $k=8$ is shown in the figure on the top right. We have shown the charge densities in the figures below their corresponding effective potentials. but heights of the peaks show a change in trend. We notice that the peaks corresponding to higher $N$ are gradually smaller for $k=1,2$ and 4 . In the inset we see that the peaks are either equal in height or gradually becomes larger for larger $N$. This can in part be due to the fact that the level spacings are increasingly larger for higher single particle states as the shape of the confinement is changed from parabolic confinement to square well confinement.

From both figures 1 and 2 one can infer that as we increase the number of electrons, there is a gradual decrease in the capacitive energy. This can be explained by looking at figure 3: when the number of electrons inside the QD (e.g. for $k=2$ ) is increased, the effective potential becomes relatively more flat so electrons find more space to move inside the dot i.e. they are less confined. Hence capacitive energy decreases gradually. Similar explanation also applies for the change in the height of the peaks. Since energy levels come down because of the lesser confinement, the height of the peaks decreases.

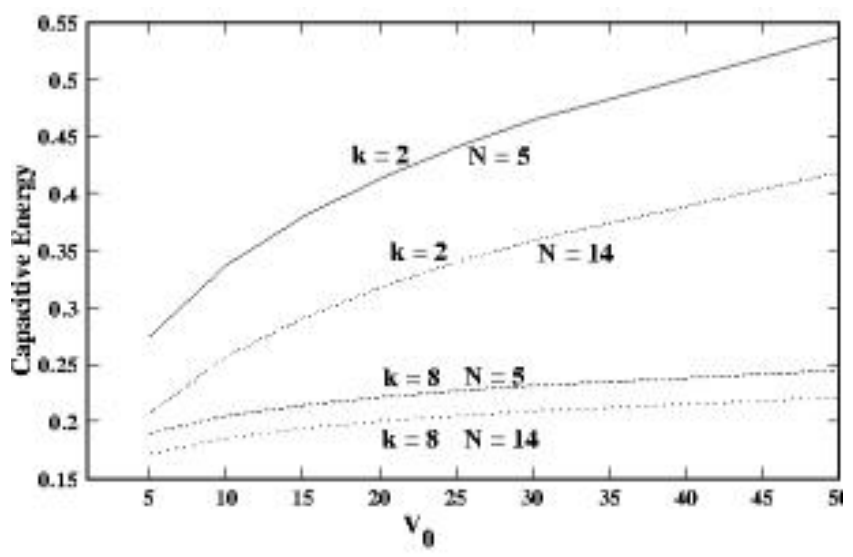

Figure 4. Capacitive energy is shown as a function of the depth of the external potential $V_{0}$ for $k=2$ and 8 and $N=5$ and 14. We observe that the capacitive energy increases as $V_{0}$ is increased and saturates at higher potential value.

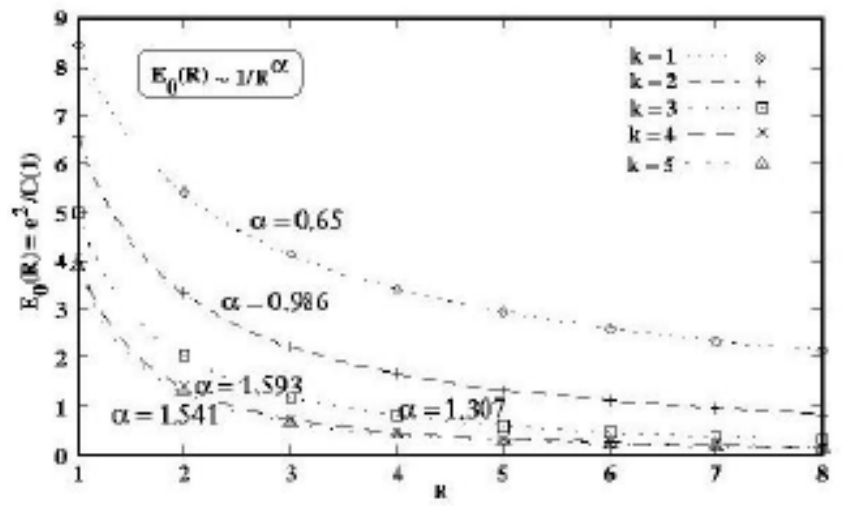

Figure 5. The ground state energy, $E_{0}$, of a single electron system is depicted as a function of size $(R)$ of a QD for different values of potential shape index, $k$. 


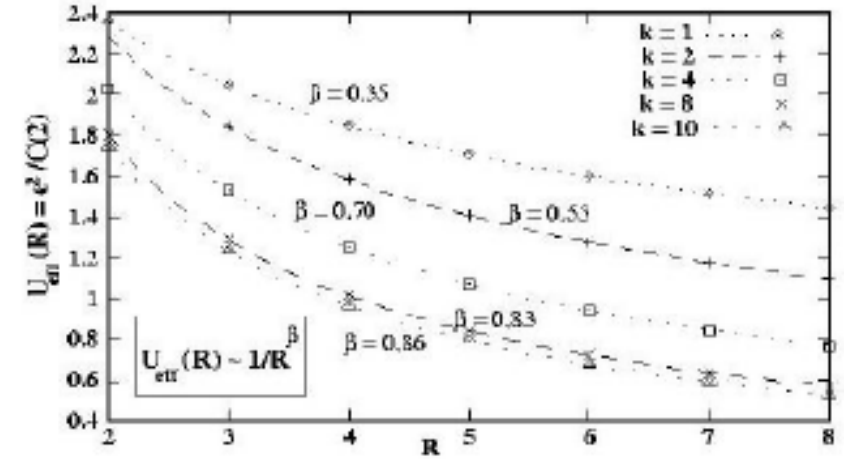

Figure 6. The effective electron-electron interaction energy for a two-electron system, $U_{\text {eff }}$, is depicted as a function of size $(R)$ of a QD for different values of potential shape index, $k$.

For large $k$ e.g. $k=10$, as the value of $N$ increases the effective potential develops a small valley near the surface (boundary) of the QD. The depth of the valley gradually increases as $N$ is increased. As a result charge density near the surface increases with the number of electrons. It seems as if the charges are trapped inside these valleys leading to enhanced confinement as the total number of charges are increased and the energy levels are pushed up.

In figure 4, we have depicted the capacitive energy as a function of the barrier height, $V_{0}$ for $N=5$ and 14. This is shown for two potential shapes, $k=2$ and 8 for size, $R=8$. As expected the capacitive energy is larger when the confinement is larger. When the barrier height is larger, then the charge leakage out of barrier is smaller, hence more charge is confined within the barrier. This leads to a larger charge density inside the QD and hence larger interaction energy or charging energy for a larger barrier. However, as the barrier height is increased further, the capacitive energy saturates to a fixed value.

In figure 5, we have depicted the ground state energy, $E_{0}(R)$ for QD with a single charge as a function of size $(R)$ and different $k$. We fit each set of data for a given $k$ to the following function

$$
f(R)=\frac{A}{R^{\alpha}} .
$$

The ground state energy, $E_{0} \sim 1 / R^{\alpha}$. Within quantum confinement model (QCM), $E_{0}$ is predicted to be $\sim 1 / R^{2}$. Notice, this is the case when $k$ is large $(k \geq 5)$, i.e. when quasi square well is considered. Most calculations for Coulomb blockade have been reported with a quasi-harmonic confinement $(k=2)$. We have obtained $1 / R$ dependence of $E_{0}$ for this case.

A similar exercise for capacitive energy, $e^{2} / C(N)$ is carried for $N=2,4,6,8$ and 10 . The plot is shown in figure 6 . The shape dependence of electron-electron inter-
Table 1. The dependence of the exponents, $\alpha$ and $\beta$, on the shape of the potential, indexed by $k$.

\begin{tabular}{rll}
\hline$k$ & \multicolumn{1}{c}{$\alpha$} & \multicolumn{1}{c}{$\beta$} \\
\hline 1 & 0.65 & 0.354 \\
2 & 0.986 & 0.528 \\
4 & 1.307 & 0.698 \\
8 & 1.541 & 0.831 \\
10 & 1.593 & 0.863 \\
12 & 1.627 & 0.885 \\
14 & 1.650 & 0.901 \\
16 & 1.667 & 0.913 \\
20 & 1.689 & 0.929 \\
80 & 1.767 & 0.967 \\
\hline
\end{tabular}

action energy is clearly reflected in the above figure. We fit these data to the following function

$$
U_{\text {eff }}(k, R)=\frac{B}{R^{\beta}} .
$$

The interaction energy, $U_{\text {eff }} \sim 1 / R^{\beta}$. Once again we observe that the generally held notion of electron-electron interaction energy $\sim 1 / R$ is true only for a quasisquare confinement $(k \geq 5)$. For the quasi-harmonic confinement $(k=2)$, $\beta$ is found to be $\approx 1 / 2$.

We have performed similar exercises for Coulomb and exchange-correlation energies and found that they exhibit the same scaling laws as the one for the capacitive energy described above.

\section{Discussion}

The size dependence of level spacing and electron-electron interaction energy is shown to be dependent on the shape of the confining potential. It has been a general belief that the level spacing varies as $\sim 1 / R^{2}$ and the electron-electron interaction energy varies as $\sim 1 / R$. Through these calculations, we show that such claims are erroneous when a harmonic confinement is used. We can see in figure 5 (also see table 1) that $\alpha$ is 1 when $k=2$. Similarly the capacitive energy (i.e. charging energy) does not go as $\sim 1 / R$ as has been the general belief, rather it varies as $1 / R^{1 / 2}$ for $k=2$ (figure 6). Hence a simple extension of quantum confinement model, where a quadratic dependence of energy levels on size is expected, may not be applicable. That outcome is a direct result of choosing a particular shape, a square well, of the potential. We have shown that the shape of the potential is strongly reflected in the size dependence of electronelectron interaction and level spacing. Also we have attempted to compare our calculations with experimental results on CdSe QDs (Klein et al 1996, 1997). Given the uncertainties in the experiment and the nature of our calculations (e.g. the LDA within the effective mass theory) detailed numerical agreement with experimental values must not be expected. However, it is heartening to note 
that we do find certain agreements between the electron and hole addition energies. A curious aspect is that the appropriate barrier for electron is quasitriangular, while for a hole it might well be quasisquare.

\section{References}

Ashoori R C 1996 Nature 379413

Harbola M K and Sahni V 1989 Phys. Rev. Lett. 62489
Iafrate G J et al 1995 Phys. Rev. B52 10737

Klein D et al 1997 Nature 389699

Klein D L et al 1996 Appl. Phys. Lett. 773613

Kohn W and Sham L J 1965 Phys. Rev. 140 A1133

Macucci M et al 1995 J. Appl. Phys. 773267

Macucci M et al 1997 Phys. Rev. B55 R4879

Singh M et al 2000 Int. J. Mod. Phys. B14 1753 and the references therein

Tarucha S et al 1996 Phys. Rev. Lett. 773613 